\title{
UNCOUPLED MULTI-LAYER MODEL FOR. THE TRANSFER OF SENSIBLE AND LATENT HEAT FLUX DENSITIES FROM VEGETATION
}

\author{
J. CHEN* \\ Department of Theoretical Production Ecology, Agricultural University, \\ Bornsesteeg 65, 6708 PD Wageningen. The Netherlands
}

(Received in final form 7 November, 1983)

\begin{abstract}
The sensible heat flux density $C$ and the latent heat flux density $\lambda E$ are coupled in the case of a multi-layer model of vegetation. Therefore two linearly independent combinations of $C$ and $\lambda E$, the enthalpy flux density $H$ and the saturation heat flux density $J$, are introduced. Two electrical analogues, for $H$ and $J$, are designed. They are equivalent to the resistance scheme for $C$ and $\lambda E$, but uncoupled. Penman's formulas for $C$ and $\lambda E$, which are applicable only to single-layer models, can be expressed equivalently in terms of $H$ and $J$. This version of Penman's formulas can be extended easily to multi-layer canopies.
\end{abstract}

\section{Introduction}

Transpiration from a crop stand has been and is still being extensively studied, both experimentally and theoretically, because of its importance to agriculture as well as to meteorology. As to the theoretical work, there are two different approaches: single-layer models and multi-layer models. Both are well developed. In the single-layer models, a crop stand is treated as one equivalent surface with a mean surface temperature, an equivalent resistance, which is often called canopy resistance, and an equivalent surface boundary-layer resistance. In the multi-layer models, the profiles of all relevant variables - not only above but also within canopies - are simulated.

For single-layer models, Penman's formulas (Penman, 1948) are the most frequently used equations for determining sensible and latent heat flux densities from an evaporato ing surface, based on the energy balance approach.

The energy balance method can also be used in the multi-layer models. The unknown variables are the temperatures and humidities of each layer, and the sensible and latent heat flux densities at different levels within the canopy. Unfortunately, the equations for sensible and latent heat flux densities are coupled, so that explicit expressions for canopy latent and sensible heat flux densities have not been developed.

Although single-layer models may not be adequate for many cases, they have been widely used because of their simplicity. Multi-layer models should be more useful if analytical solutions can be offered for their steady-state flux densities. Shuttleworth (1976) derived a so-called combination equation in an attempt to obtain a unified model describing single- and multi-layer models.

In the present paper, the multi-layer model developed by Goudriaan and Waggoner (1972) is examined. Then two linearly independent combinations of sensible and latent

* Permanent address: Institute of Plant Physiology, Academia, Sinica, 300 Fenglin Road, Shanghai, China. 
heat flux densities are introduced. Using the enthalpy flux density $H$ and the saturation heat flux density $J$ as two new variables, the equations are uncoupled.

Two electrical analogues, for $H$ and $J$, are designed. Based on the uncoupled electrical analogues, analytical solutions can be found.

\section{Theory}

\subsection{COUPLED MULTI-LAYER MODEL}

Agrometeorologists are interested not only in the sensible and latent heat flux densities above a crop canopy, but also in the profiles of temperature and humidity within the canopy. To simulate these profiles, single-layer models are no longer applicable. One has to divide the whole canopy into several layers, and define the leaf temperature, air temperature and humidity, and the sensible and latent heat flux densities in each layer as state variables.

Because the heat capacity of the air in the free space within the canopy and that of the leaves are rather small for a mean state over a relatively long period, one hour say, the heat storage term can be ignored in the energy balance equation. For every layer, therefore, the net radiation absorbed can be considered to be equal to the sum of the sensible and latent heat flux densities. These energy balance equations together with the relationships between flux densities and relevant driving forces, using the analogy of the electrical circuit theory, constitute a closed set of equations for all unknown variables.

This method was used by Waggoner et al. (1969) and Goudriaan and Waggoner (1972). The electrical analogue shown in Figure 1 is based on these two papers, but the notations for the variables and the symbols for the potential and current sources are adapted to standard usage.

The driving force (potential source) above the canopy for sensible heat flux density is $\rho c_{p} T_{a, 0}$, in which $\rho c_{p}$ is the volumetric heat capacity of air and $T_{a, 0}$ is the air temperature at the reference height. The driving force above the canopy for latent heat flux density is $\left(\rho c_{p} / \gamma\right) e_{a, 0}$, where $\gamma$ is the psychometric constant and $e_{a, 0}$ is the water vapour pressure of the air at the reference height. In addition to these two potential sources, there is a current source for each layer. This is the net radiation absorbed within the layer, denoted as $S_{i}$, where $i$ specifies the layer index.

The sensible and latent heat flux densities supplied by the layer $i$ are denoted by $C_{i}$ and $\lambda E_{i}$, respectively. The corresponding resistances are specified by $r_{H, i}$ and $r_{V, i^{*}}$ The resistances in the vertical direction due to turbulent exchange are represented by the $R_{i}^{\text {' }}$, which are assumed to be the same for both the sensible and latent heat transfers.

Based on this electrical analogue, the following equations can be obtained:

$$
\begin{aligned}
& C_{i}+\lambda E_{i}=S_{i} \\
& C_{i}=\rho c_{p}\left(T_{L, i}-T_{a, i}\right) / r_{H, i} \\
& \lambda E_{i}=\left(\rho c_{p} / \gamma\right)\left(e_{s}\left(T_{L, i}\right)-e_{a, i}\right) / r_{V, i} \\
& e_{s}\left(T_{L, i}\right)=e_{s}\left(T_{a, i}\right)+\Delta\left(T_{L, i}-T_{a, i}\right) .
\end{aligned}
$$




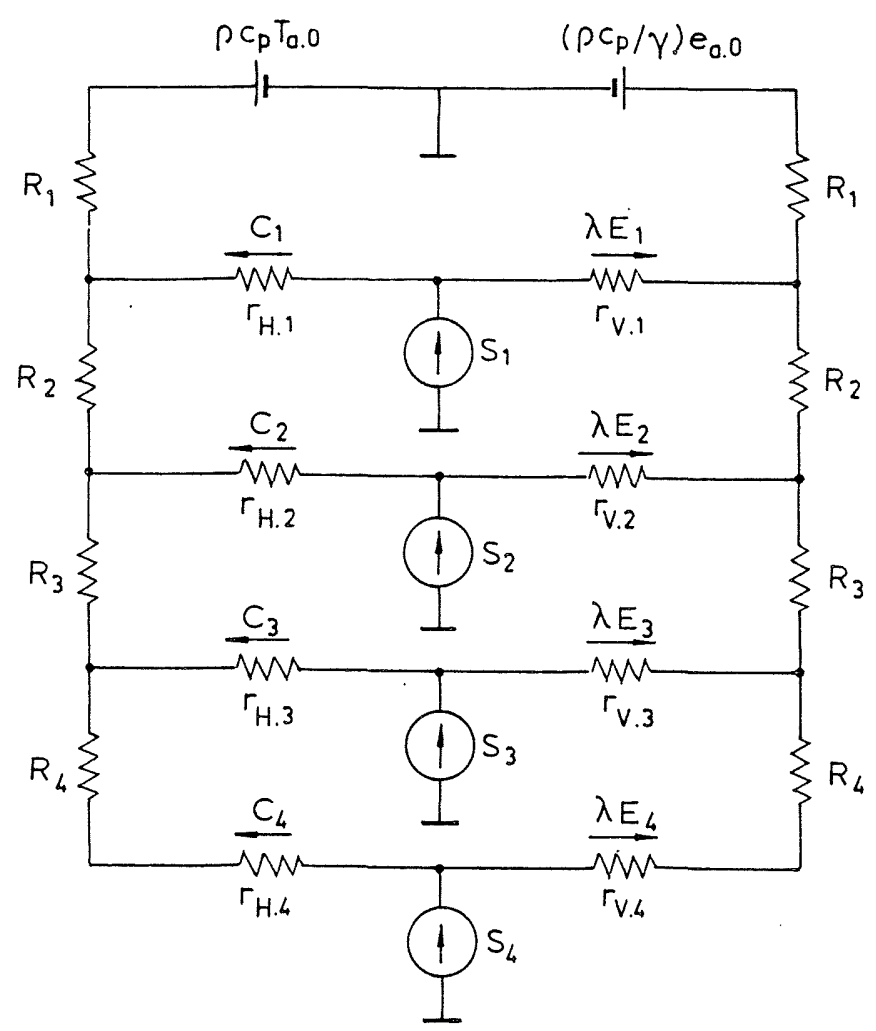

Fig. 1. Electrical analogue for sensible and latent heat flux densities. For meaning of symbols, see the text.

Equation (1) is the energy balance equation. Equations (2) and (3) are the analogues of Ohm's law for the sources of the sensible and latent heat flux densities. The sensible heat flux density originates from the leaf surfaces, so that the leaf temperature $T_{L, i}$ is introduced. $T_{a, i}$ is the temperature of the air surrounding the leaves. $r_{H, i}$ consists of only the leaf boundary-layer resistance for heat transfer. The latent heat flux density originates from the substomatal cavities. The air within the cavities is assumed to be saturated by water vapour, so that the vapour pressure there can be determined as the saturated vapour pressure at the corresponding leaf temperature $e_{s}\left(T_{L, i}\right)$. The variable $e_{a, i}$ is the vapour pressure of the air surrounding the leaves. The resistance for the vapour transfer from the substomatal cavities to the surrounding free space within the canopy, $r_{V, i}$, is composed of two parts: the stomatal resistance and the leaf boundary-layer resistance.

Due to the different diffusion coefficients of vapour and heat in air, the leaf boundarylayer resistance for latent heat is slightly different from $r_{H, i}$. It is often convenient to define the stomatal resistance $r_{s}$ as

$$
r_{s}=r_{V}-r_{H}
$$


Equation (4) is the linearized saturated vapour pressure versus temperature curve, which is in fact more or less exponential, and $\Delta$ is its slope determined at a proper temperature, $T_{p}$.

To complete the system of equations, Ohm's law can be applied in the vertical direction:

$$
\begin{array}{ll}
\sum_{j=n}^{i} C_{j}=\rho c_{p}\left(T_{a, i-1}-T_{a, i}\right) / R_{i} & (i=1 \text { to } n) \\
\sum_{j=n}^{i} \lambda E_{j}=\left(\rho c_{p} / \gamma\right)\left(e_{a, i-1}-e_{a, i}\right) / R_{i} & (i=1 \text { to } n)
\end{array}
$$

where $n$ specifies the total number of layers.

The driving forces in the vertical direction for sensible and latent heat flux densities are, respectively, the differences in $\rho c_{p} T_{a}$ and $\rho c_{p} e_{a} / \gamma$ between layers, while the flux densities through $R_{i}$ are composed of all sources from layers $i$ to $n$, so that the summation is carried out on the left-hand sides of Equations (6) and (7).

Equations (1) through (4) and (6), (7) constitute a closed set of equations. Being coupled, they have to be solved simultaneously. A matrix method was developed by Waggoner et al. (1969). Although sophisticated software for solving matrix problems is available, explicit analytical solutions are needed to simplify the application of this theory and to provide a more direct physical insight into canopy behaviour. The idea is to make the equations uncoupled by introducing two linearly independent combinations of $C$ and $\lambda E$.

\subsection{UNCOUPLING}

One of the two required combinations of $C$ and $\lambda E$ is straightforward. It follows from Equation (1) that if a new variable $H$, called the enthalpy flux density, is defined as:

$$
H=C+\lambda E
$$

the source for $H$ from layer $i$, following Equation (1), can be written as:

$$
H_{i}=S_{i} \text {. }
$$

Because the net radiation absorbed within each layer is a known variable, $H_{i}$ can be obtained immediately.

The driving force for the enthalpy flux density in the vertical direction is the difference in $\rho c_{p}\left(T_{a}+e_{a} / \gamma\right)$ between layers, a combination of the driving forces for sensible and latent heat flux densities, following the definition of $H$. The term $T_{a}+e_{a} / \gamma$ is often called the equivalent temperature of air, denoted by $T e_{a}$ :

$$
T e_{a}=T_{a}+e_{a} / \gamma \text {. }
$$

The problem is to find another combination.

It follows from Equations (1) through (4) that $C_{i}$ and $\lambda E_{i}$ can be rewritten as:

$$
C_{i}=\left(\gamma r_{V, i} S_{i}-\rho c_{p} D_{i}\right) /\left(\Delta r_{H, i}+\gamma r_{V, i}\right)
$$




$$
\lambda E_{i}=\left(\Delta r_{H, i} S_{i}+\rho c_{p} D_{i}\right) /\left(\Delta r_{H, i}+\gamma r_{V, i}\right)
$$

where $D_{i}$ is the water vapour pressure deficit of the air in layer $i$ :

$$
D_{i}=e_{s}\left(T_{a, i}\right)-e_{a, i} \text {. }
$$

To find the other combination, it can be noticed that Equations (11) and (12) only contain one property of the air in the form of the vapour pressure deficit, $D_{i}$. Therefore the flux density driven by the difference in $D$ between layers, should be the desired combination of the sensible and latent heat flux densities.

The saturated vapour pressure at air temperature, $e_{s}\left(T_{a}\right)$, can be expressed as:

$$
e_{s}\left(T_{a}\right)=e_{s}\left(T_{p}\right)+\Delta\left(T_{a}-T_{p}\right)
$$

where $T_{p}$ is the properly chosen temperature for evaluating $\Delta$, as mentioned before.

Equation (13) can be rewritten as:

$$
D_{i}=\Delta T_{a, i}-e_{a, i}+e_{s}\left(T_{p}\right)-\Delta T_{p} .
$$

By taking the difference of $D_{i}$ between layers $i-1$ and $i$, the constant $e_{s}\left(T_{p}\right)-\Delta T_{p}$ in Equation (15) is eliminated. A subsequent multiplication of both sides with $\left(\rho c_{p} / \Delta\right) / R_{i}$ yields:

$$
\begin{aligned}
\left(\rho c_{p} / \Delta\right)\left(D_{i-1}-D_{i}\right) / R_{i}= & \rho c_{p}\left(T_{a, i-1}-T_{a, i}\right) / R_{i}- \\
& (\gamma / \Delta)\left(\rho c_{p} / \gamma\right)\left(e_{a, i-1}-e_{a, i}\right) / R_{i} .
\end{aligned}
$$

Referring to Equations (6) and (7) shows that the right-hand side of Equation (16) can be written as $C_{i}^{*}-(\gamma / \Delta) \lambda E_{i}^{*} . C_{i}^{*}$ and $\lambda E_{i}^{*}$ denote the cumulative sensible and latent heat flux densities above layer $i$, which are the sums of the relevant sources from layers $i$ through $n$ as expressed on the left-hand side of Equations (6) and (7). The left-hand side of Equation (16) represents a new cumulative flux density denoted now by $J_{i}^{*}$ :

$$
J_{i}^{*}=\sum_{j=n}^{i} J_{j}=\left(\rho c_{p} / \Delta\right)\left(D_{i-1}-D_{i}\right) / R_{i}
$$

where $J_{j}$ is the source term from layer $j$. Therefore the desired combination of the sensible and latent heat flux densities is:

$$
J=C-(\gamma / \Delta) \lambda E
$$

which is valid for both $J_{i}^{*}$ and $J_{i}$.

It follows from Equations (11), (12), and (f8) that the source $J_{i}$ is:

$$
J_{i}=\frac{-\left(\rho c_{p} / \Delta\right) D_{i}}{r_{H, i}+\alpha r_{s, i}}+\frac{S_{i}}{\left(1+r_{H, i} / \alpha r_{s, i}\right)}
$$

where $r_{s}$ is the stomatal resistance defined by Equation (5), and $\alpha$ is defined as:

$$
\alpha=\gamma /(\gamma+\Delta) \text {. }
$$




\subsection{UNCOUPLED ELECTRICAL ANALOGUES FOR $H$ AND $J$}

For the required electrical analogue for $H$, a potential source above the canopy can be specified as $\rho c_{p} T e_{a, 0}$, in which $T e_{a, 0}$ is the equivalent temperature of the air at the reference height. The current sources are given by Equation (9) for each layer.

"The electrical analogue is simple, as is shown in Figure 2. The meaning of the symbols is the same as those in Figure 1. Now $H_{i}^{*}$ specifies the cumulative flux density above layer $i$, which is the sum of the sources $H_{i}$ from layers $i$ through $n . H_{i}^{*}$ is positive upward. The change of usage is necessary for obtaining a simpler form of solution later. It is obvious that the $H_{i}^{*}$ 's are determined only by the current sources while the potential source gives no contribution to the $H_{i}^{* \prime}$.

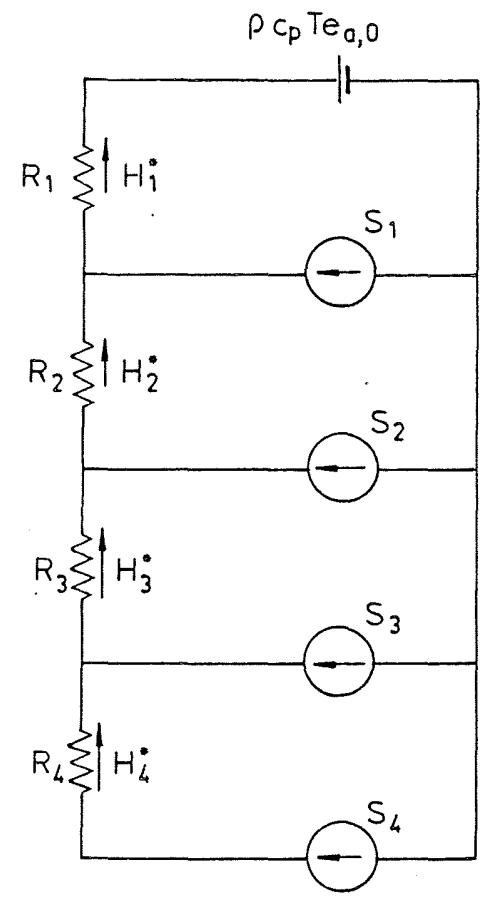

Fig. 2. Electrical analogue for enthalpy flux density $H$. For meaning of symbols, see the text.

Similar to the electrical analogue for the enthalpy flux density $H$, a potential source above the canopy for $J$ can be specified as $\left(\rho c_{p} / \Delta\right) D_{0}$, in which $D_{0}$ is the vapour pressure deficit of the air at the reference height.

The role of the current sources requires more consideration. First, a diagram can be designed for a single layer. Inspection of Equation (19) indicates that $J_{i}$ is composed of two parts. The first term on the right-hand side of Equation (19) is due to the potential source $\left(\rho c_{p} / \Delta\right) D_{i}$. The diagram can be easily designed, as is shown in Figure 3a. The second term on the right-hand side of Equation (19) is caused by the current source $S_{i}$ (for the soil surface, the heat flux into the soil $G$ should be subtracted from $S_{i}$ ). The diagram obtained is shown in Figure $3 \mathrm{~b}$. The diagrams in Figure $3 \mathrm{a}$ and $\mathrm{b}$ can be 

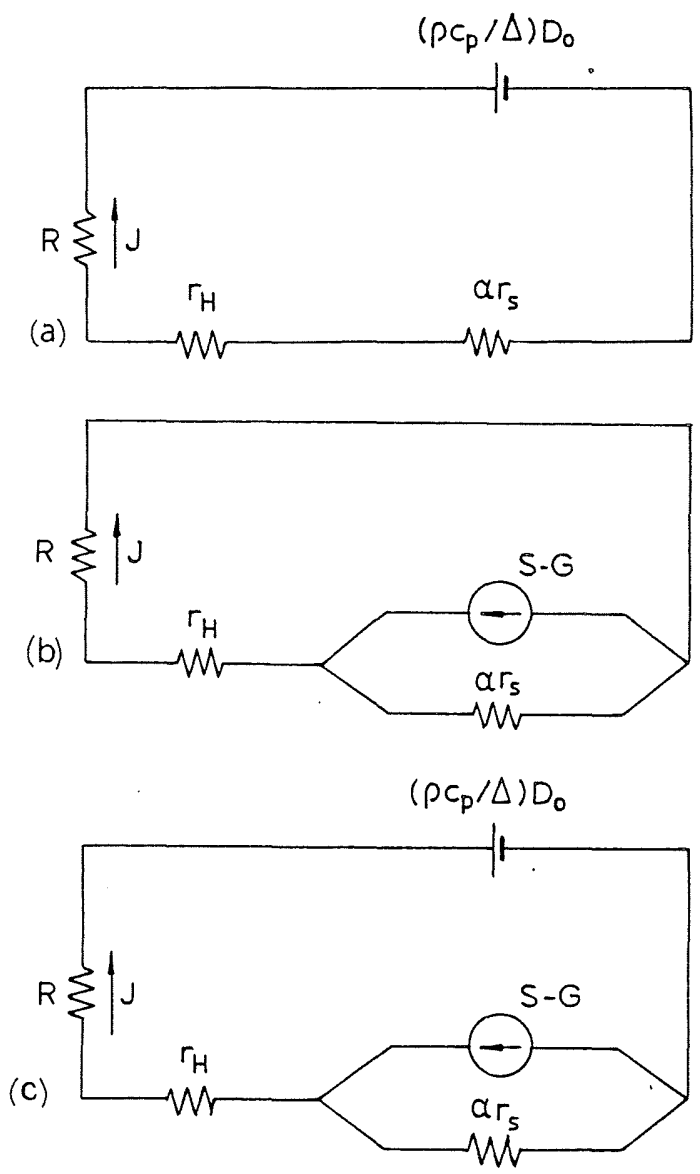

Fig. 3. Electrical analogues for $J$ for one sublayer. (a) For the potential source; (b) for the current source; (c) combined.

combined, according to the superposition theorem in electrical theory, as shown in Figure 3c. The electrical analogue for the whole canopy can be obtained, based on that for the single layers (Figure 4).

\subsection{SOlUtions FOR THE TOTAL FLUX DENSITIES $H_{1}^{*}$ AND $J_{1}^{*}$}

The total flux density $H_{1}^{*}$ above the canopy is simply:

$$
H_{1}^{*}=\sum_{j=1}^{n} S_{j}
$$

while that of $J_{1}^{*}$ has to be determined from Figure 4.

For simplicity, only four layers are assumed, but the solutions can be easily extended to the general case. Under the notation presently used, $J_{i}^{*}$ is now cumulative to each layer $i$, hence $J_{i}=J_{i}^{*}-J_{i+1}^{*}$, and Equation (19) becomes:

$$
r_{i}\left(J_{i}^{*}-J_{i+1}^{*}\right)=-\left(\rho c_{p} / \Delta\right) D_{i}+\alpha r_{s, i} S_{i} \quad(i=1 \text { to } 4)
$$




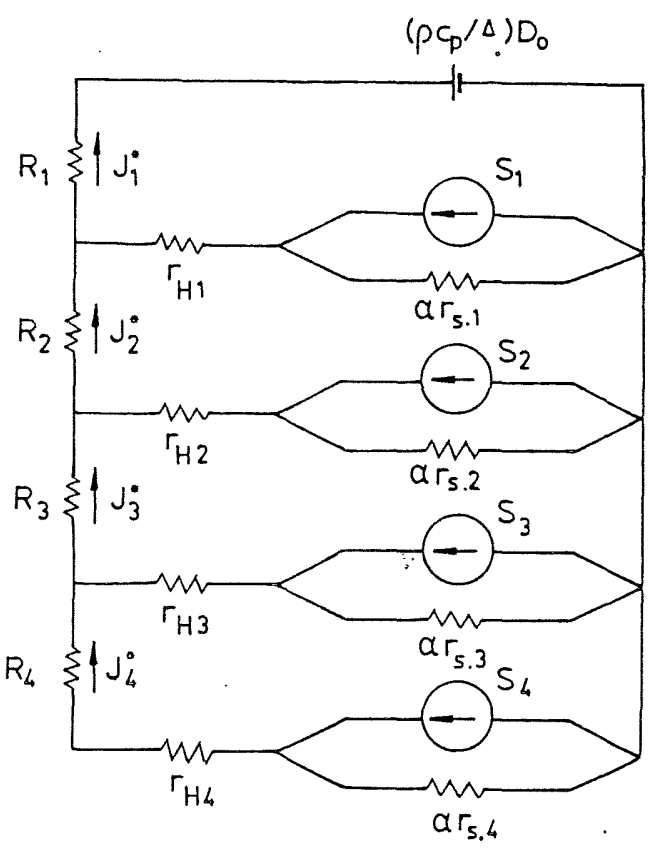

Fig. 4. Electrical analogue for saturation heat flux density $J$. For meaning of symbols, see the text.

where $J_{5}^{*}$ is defined as zero, and $r_{i}$ is defined as:

$$
r_{i}=r_{H, i}+\alpha r_{s, i} \text {. }
$$

The other four equations can be obtained for each $R_{i}$ in the vertical direction:

$$
R_{i} J_{i}^{*}=\left(\rho c_{p} / \Delta\right)\left(D_{i}-D_{i-1}\right) \quad(i=1 \text { to } 4) .
$$

Equations (22) and (24) represent eight equations for eight unknown variables: four $J_{i}^{*}$ 's and four $D_{i}$ 's, so that this is a closed system.

Substituting Equation (22) into Equation (24) and rearranging gives four equations for the $J_{i}^{*}$ 's:

$$
\begin{array}{rlrl}
\left(1+R_{1} / r_{1}\right) J_{1}^{*}- & J_{2}^{*} & & =b_{1} \\
\left(R_{1} / r_{2}\right) J_{1}^{*}+\left(1+R_{2} / r_{2}\right) J_{2}^{*}- & J_{3}^{*} & =b_{2} \\
\left(R_{1} / r_{3}\right) J_{1}^{*}+ & \left(R_{2} / r_{3}\right) J_{2}^{*}+\left(1+R_{3} / r_{3}\right) J_{3}^{*}- & J_{4}^{*} & =b_{3} \\
\left(R_{1} / r_{4}\right) J_{1}^{*}+ & \left(R_{2} / r_{4}\right) J_{2}^{*}+ & \left(R_{3} / r_{4}\right) J_{3}^{*}+\left(1+R_{4} / r_{4}\right) J_{4}^{*} & =b_{4}
\end{array}
$$

where

$$
b_{i}=\left[-\left(\rho c_{p} / \Delta\right) D_{0}+\alpha r_{s, i} S_{i}\right] / r_{i} .
$$


$J_{1}^{*}$ can be solved by using Cramer's rule:

$$
J_{1}^{*}=\frac{\left|\begin{array}{rrrr}
b_{1} & -1 & 0 & 0 \\
b_{2} & 1+R_{2} / r_{2} & -1 & 0 \\
b_{3} & R_{2} / r_{3} & 1+R_{3} / r_{3} & -1 \\
b_{4} & R_{2} / r_{4} & R_{3} / r_{4} & 1+R_{4} / r_{4}
\end{array}\right|}{\left|\begin{array}{rrrr}
1+R_{1} / r_{1} & -1 & 0 & 0 \\
R_{1} / r_{2} & 1+R_{2} / r_{2} & -1 & 0 \\
R_{1} / r_{3} & R_{2} / r_{3} & 1+R_{3} / r_{3} & -1 \\
R_{1} / r_{4} & R_{2} / r_{4} & R_{3} / r_{4} & 1+R_{4} / r_{4}
\end{array}\right|} .
$$

By denoting the determinant in the denominator in Equation (27) as $A_{0}$, and defining the sub-determinants as:

$$
\begin{aligned}
& A_{1}=\left|\begin{array}{rrr}
1+R_{2} / r_{2} & -1 & 0 \\
R_{2} / r_{3} & 1+R_{3} / r_{3} & -1 \\
R_{2} / r_{4} & R_{3} / r_{4} & 1+R_{4} / r_{4}
\end{array}\right| \\
& A_{2}=\left|\begin{array}{rr}
1+R_{3} / r_{3} & -1 \\
R_{3} / r_{4} & 1+R_{4} / r_{4}
\end{array}\right| \\
& A_{3}=1+R_{4} / r_{4} \\
& A_{4}=1,
\end{aligned}
$$

Equation (27) can be written in a concise form by unfolding the numerator according to the column $b_{i}$ :

$$
\begin{aligned}
J_{1}^{*} & =\sum_{j=1}^{n}\left(A_{j} / A_{0}\right) b_{j} \\
& =\sum_{j=1}^{n}\left(A_{j} / A_{0}\right)\left[-\left(\rho c_{p} / \Delta\right) D_{0} / r_{j}+\left(\alpha r_{s, j} / r_{j}\right) S_{j}\right] .
\end{aligned}
$$

A recurrent relation between the $A_{i}^{\prime}$ s can be found. Unfolding $A_{1}$, for instance, according to the first column gives:

$$
\begin{aligned}
A_{1} & =\left(1+R_{2} / r_{2}\right) A_{2}+\left(R_{2} / r_{3}\right) A_{3}+\left(R_{2} / r_{4}\right) A_{4} \\
& =A_{2}+R_{2} \sum_{j=2}^{n} A_{j} / r_{j} .
\end{aligned}
$$




$$
A_{i}=A_{i+1}+R_{i+1} \sum_{j=i+1}^{n}\left(A_{j} / r_{j}\right) \quad(i=0 \text { to } n-1) .
$$

The profiles of $J$ can also be obtained, as well as those of air temperature, vapour pressure and leaf temperature. Because these profiles are calculated from the explicit expressions, the computing time is greatly reduced, so that the calculation can be executed on a microcomputer. But this subject will be left for another article.

\subsection{AN EQUIVALENT EXPRESSION FOR PENMAN'S FORMULAS}

As mentioned above, Penman's formulas are often used for calculating the sensible and latent heat flux densities from an evaporating surface:

$$
\begin{aligned}
& C=\frac{\gamma^{*}(S-G)-\rho c_{p} D_{0} /\left(R+r_{H}\right)}{\Delta+\gamma^{*}} \\
& \lambda E=\frac{\Delta(S-G)+\rho c_{p} D_{0} /\left(R+r_{H}\right)}{\Delta+\gamma^{*}}
\end{aligned}
$$

where $G$ is the heat flux density into the soil and $\gamma^{*}$ is the apparent psychrometric constant, defined as:

$$
\gamma^{*}=\gamma\left(R+r_{V}\right) /\left(R+r_{H}\right) .
$$

The problem is that no equivalent expressions exist for $C$ and $\lambda E$ in the multi-layer model.

By using the definitions of $H$ and $J$, Equations (8) and (17), however, Penman's formulas can also be expressed in terms of $H$ and $J$ :

$$
\begin{aligned}
& H=S-G \\
& J=\frac{\alpha r_{s}(S-G)-\left(\rho c_{p} / \Delta\right) D_{0}}{R+r_{H}+\alpha r_{s}} .
\end{aligned}
$$

These equivalent expressions for Penman's formulas are in fact only special forms of Equations (21) and (32) as can be seen as follows.

For the single-layer model, $n=1, S_{j}$ becomes $S-G$, so that Equation (21) turns out to be Equation (38). In this case $A_{1}=1$, and $A_{0}=1+R /\left(r_{H}+\alpha r_{s}\right)$, so that Equation (32) becomes the same as Equation (39). In fact, $C$ and $\lambda E$ can be expressed in terms of $H$ and $J$ :

$$
\begin{aligned}
& C=\alpha H+(1-\alpha) J \\
& \lambda E=(1-\alpha)(H-J) .
\end{aligned}
$$

The familiar Penman equations (35) and (36) are obtained by substituting Equations (38) and (39) into (40) and (41) and simplifying. The equivalent Penman's formulas in 
terms of $H$ and $J$, Equations (38) and (39), are more preferable; because for both single-layer and multi-layer models, the same formulas can be used.

As a matter of fact, Equations (21) and (32) provide a bridge between the single-layer and multi-layer models, from which several conclusions, particularly for the canopy resistance $r_{c}$, can be drawn. This is again, however, beyond the scope of the present paper, in which only the theoretical basis of the method is presented.

\subsection{Physical Significance of $J$}

The flux densities $H$ and $J$ were introduced during the mathematical process of uncoupling. But it can be seen that they have clear physical meanings. The physical meaning of $H$ is obvious, viz., the total heat or enthalpy flux density. On the contrary, the physical meaning of $J$ is not immediately clear and is exposed now.

The Bowen ratio $\beta$ is defined as the ratio of sensible to latent heat flux densities. It can be expressed in terms of $H$ and $J$ :

$$
\beta=C / \lambda E=[(\gamma / \Delta) H+J] /(H-J) .
$$

This equation shows that a unique relationship exists between $\beta$ and $J$, as long as $H$ is fixed. The larger the $J$, the larger the Bowen ratio will be. It can be seen that:

$$
\begin{array}{lll}
\beta=\gamma / \Delta & \text { when } & J=0 \\
\beta<\gamma / \Delta & \text { when } & J<0 \\
\beta>\gamma / \Delta & \text { when } & J>0
\end{array}
$$

in which the value of $\gamma / \Delta$ is often called the critical value of the Bowen ratio (Monteith, 1973). Likewise $J=0$ can also be called a critical value.

According to the preceding derivation, the flux density $J$ is driven by the gradient of $D$. Therefore when $J$ equals zero, the gradient of vapour pressure deficit is zero, and vice versa. In this case, $\lambda E$ is equal to $(1-\alpha) H$ or

$$
\lambda E=(1-\alpha)(S-G)
$$

which is often called the equilibrium evaporation rate (Priestley and Taylor, 1972).

In addition to the three classical flux densities of enthalpy, sensible heat and latent heat, it is proper to emphasize the role of $J$, as a fourth flux density. Together with the driving forces (gradients) they are:

Driving force (gradient):

temperature $T$

vapour pressure $e$

equivalent temperature $T e$

vapour pressure deficit $D$
Flux density:

sensible heat $C$

latent heat $\lambda E$

enthalpy $H$

saturation heat $J$.

Noting the important role of the vapour pressure deficit in evapotranspiration and the 
clear physical meaning of the related flux density $J$, it can be realized that $J$ is not merely a mathematical device. It seems proper to give it a name. Here $J$ is called the saturation heat flux density.

\section{Discussion and Conclusions}

The saturation heat flux density $J$ is equivalent to $\psi_{2}=-\Delta C+\gamma \lambda E$, derived by McNaughton (1976) in his two-dimensional and single-layer model for evaporation and advection. Perrier (1976) has also derived a second-order differential equation for the so-called saturation temperature deficit $Y=T-T d$, where $T d$ is the dew point temperature. Both their derivations are based on a continuous model in the vertical direction rather than on a discrete multi-layer model.

Because the resulting second-order differential equation cannot be solved analytically and a numerical solution based on a difference method has to be used, it is more straightforward to derive the equations for $J$ directly from a multi-layer model. Based on these equations for the discrete model, an electrical analogue, which gives a clearer picture, can be easily designed.

In the present paper, the quantity $J$ is introduced, based on the linearized saturated vapour pressure versus temperature curve. This is only an approximation. Inspection of the saturated vapour pressure curve shows that within a 10-deg temperature interval, the error caused by the linearization is rather small. Calculations show that for the intervals of $10-20,20-30$, and $30-40^{\circ} \mathrm{C}$, the largest relative errors are, respectively, $4.3,3.0$, and $1.8 \%, \Delta$ being evaluated at the mid-points of the intervals. For a larger interval of $15-35{ }^{\circ} \mathrm{C}$, however, the relative error reaches $13.5 \%$. This temperature interval is determined in practice by the difference between the highest leaf temperature and the lowest air temperature following Equation (4). Under most field conditions, this temperature difference is not expected to exceed $10^{\circ} \mathrm{C}$, so that linearization of the saturated vapour pressure curve is feasible.

The substitution of $D / \Delta$ by the saturation temperature deficit $Y$ extends unnecessarily the temperature interval. The lowest air temperature now is replaced by the lowest dew point temperature. The extension depends on the value of $D$, or more precisely, on the relative humidity $h$. Calculations show that as a rule of thumb, $h=0.5$ is equivalent to $Y=10^{\circ} \mathrm{C}$. Thus the substitution of $Y$ for $D / \Delta$ introduces an additional error, which becomes substantial when $h$ is small.

The other basic assumption is related to the substitution of $H$ and $J$ for $C$ and $\lambda E$, viz., the similarity between the exchange coefficients of heat and water vapour. This is a rather good approximation, the conditions for its validity having been extensively studied (Monteith, 1973). Obviously, it does not hold for the transfer process in the soil. When the substitution is applied to the canopy, the heat flux density into the soil at the soil surface, $G$, should be known. If $G$ is unknown and is an output of the simulation program as in Goudriaan's model (1977), an iteration method has to be used.

The treatment in the present paper is different from that by Shuttleworth (1976). He derived his combination equation based on the redefinition of the relevant resistances, 
e.g., $r_{H}$ and $r_{V}$. The introduction of $H$ and $J$ retains all the resistances in an ordinary sense, and the electrical analogues thus developed use the concepts of the potential and current sources in a standard way.

Based on these considerations, the following conclusions can be drawn:

(a) To study transpiration from a canopy, flux densities of enthalpy $H$ and saturation heat $J$ are preferred to flux densities of sensible heat $C$ and latent heat $\lambda E$, because the resulting equations are uncoupled.

(b) The flux density $J$ is uniquely related to the Bowen ratio at each value of $H$. The equilibrium evaporation rate occurs when $J=0$.

(c) The electrical analogues for $H$ and $J$ provide a method of calculating the flux densities; the method is applicable to both single-layer models and multi-layer models. In the case of single-layer models, the derived formulas for $H$ and $J$ are another version of the familiar Penman formulas for $C$ and $\lambda E$.

\section{Acknowledgment}

This work was carried out in connection with a research program supported by the Ministry of Education and Science of the Netherlands. I gratefully acknowledge this support.

I am much indebted to Dr Goudriaan, Dr F. W. T. Penning de Vries, Prof. C. T. de Wit, Prof. D. A. de Vries, and Prof. J. L. Monteith for many helpful suggestions and discussions.

\section{References}

Goudriaan, J.: 1977, ‘Crop Micrometeorology: A Simulation Study’, Pudoc, Wageningen.

Goudriaan, J. and Waggoner, P. E.: 1972, 'Simulating both Aerial Microclimate and Soil Temperature from Observations above the Foliar Canopy', Neth. J. Agric. Sci. 20, 104-124.

McNaughton, K. G.: 1976, 'Evaporation and Advection I: Evaporation from Extensive Homogeneous Surfaces', Quart. J. Roy. Meteorol. Soc. 102, 181-191.

Monteith, J. L.: 1973, 'Principles of Environmental Physics', Edward Arnold, London.

Penman, H. L.: 1948, 'Natural Evaporation from Open Water, Bare Soil, and Grass', Proc. Roy. Soc. A194, 120.

Perrier, A.: 1976, 'Etude et essai de modélisation des échanges de masse et d'énergie au niveau des couverts végétaux', Thèse de Doctorat d'État, Université de Paris VI, avril 1976, Paris, 240 pp.

Priestley, C. H. B. and Taylor, R. J.: 1972, 'On the Assessment of Surface Heat Flux and Evaporation Using Large-Scale Parameters', Mon. Weather Rev. 106, 81-92.

Shuttleworth, W. J.: 1976, 'A One-Dimensional Theoretical Description of the Vegetation-Atmosphere Interaction', Boundary-Layer Meteorol. 10, 273-302.

Waggoner, P. E., Furnival, G. M., and Reifsnyder, W. E.: 1969, 'Simulation of the Microclimate in a Forest', Forest Sci. 15, 37-45. 